\section{An imperfect system?}

When could this elegant hypoxia-alert mechanism fail? Wang et al. suggest one potential scenario, namely during severe hypoxia. They found that when alveolar hypoxia is sufficiently intense as to decrease the blood oxygen tension to well below $30 \mathrm{mmHg}$, the endothelial depolarization that is critical to the hypoxia alert does not occur. Consequently, there is no increase in endothelial $\mathrm{Ca}^{2+}$ and, presumably, no transmission of any $\mathrm{Ca}^{2+}$-dependent warning signals to arteriolar vessels through endothelial gap junctions. A similar failure of capillary signaling could occur in conditions such as lung injury if there is endothelial damage, causing loss of endothelial gap junctional communication. Acute lung inflammation decreases lung expression of connexin 40 (7), the same connexin that alerts arterioles to alveolar hypoxia (5). In fact, the finding that HPV is abrogated in connexin 40-knockout mice (5) suggests that in lung injury, loss of signaling through endothelial gap junctions might contribute to HPV failure, and hence hypoxemia severity, in this condition.

The larger significance of these findings in lung vascular biology might relate to other capillary responses that are reported to occur after activation of voltage-depen- dent $\mathrm{Ca}^{2+}$ channels. Stoppage of lung blood flow causes endothelial membrane depolarization in lung capillaries, leading to endothelial $\mathrm{Ca}^{2+}$ entry through the voltagedependent $\mathrm{Ca}^{2+}$ channel $\alpha_{1 \mathrm{G}}$ - the same channel studied by Wang et al. - to cause release of reactive oxygen species through NADPH oxidase induction (8). The PAR-1 activator thrombin also induces endothelial $\mathrm{Ca}^{2+}$ entry through the $\alpha_{1 \mathrm{G}}$ channel to activate endothelial expression of the leukocyte adhesion receptor P-selectin in alveolar capillaries (9). Taking these diverse findings together, it would seem that voltage-activated $\mathrm{Ca}^{2+}$ entry forms a fulcrum for multiple lung vascular phenomena that could potentially coalesce into a pathogenic process. Clearly, more needs to be known as to how voltage-dependent $\mathrm{Ca}^{2+}$ entry in lung endothelium co-opts gap junctional communication to promote vascular diseases of the lung. Nevertheless, the mechanisms of hypoxia sensing now identified by Wang et al. open up possibilities for targeting voltage-gated channels and gap junctional proteins in the therapy of diseases in which loss of the protection by HPV is a known adverse factor.

\section{Acknowledgments}

The author acknowledges Sunita Bhattacharya and Mallar Bhattacharya for reading the manuscript. J. Bhattacharya is supported by NIH grants HL36024 and HL64896.

Address correspondence to: Jahar Bhattacharya, Columbia University College of Physicians and Surgeons, Lung Biology Laboratory, Department of Medicine, 630 West 168th Street, BB 8-812, New York, New York 10032, USA. Phone: 212.305.7093; Fax: 212.305.6701; E-mail: jb39@columbia.edu.

1. Sylvester JT, Shimoda LA, Aaronson PI, Ward JP. Hypoxic pulmonary vasoconstriction. Physiol Rev. 2012;92(1):367-520.

2. Weibel E. [Blood vessel anastomoses in the human lungs]. Z Zellforsch Mikrosk Anat. 1959;50:653-692.

3. Jameson AG. Diffusion of gases from alveolus to precapillary arteries. Science. 1963;139(3557):826-828.

4. Conhaim RL, Staub NC. Reflection spectrophotometric measurement of $\mathrm{O} 2$ uptake in pulmonary arterioles of cats. J Appl Physiol. 1980;48(5):848-856.

5 . Wang L, et al. Hypoxic pulmonary vasoconstriction requires connexin 40-mediated endothelial signal conduction. J Clin Invest. 2012;122(11):4218-4230.

6. Parthasarathi K, et al. Connexin 43 mediates spread of $\mathrm{Ca} 2+$-dependent proinflammatory responses in lung capillaries. JClin Invest. 2006;116(8):2193-2200.

7. Rignault S, Haefliger JA, Waeber B, Liaudet L, Feihl F. Acute inflammation decreases the expression of connexin 40 in mouse lung. Shock. 2007;28(1):78-85.

8. Wei Z, Manevich Y, Al-Mehdi AB, Chatterjee $\mathrm{S}$, Fisher AB. Ca2+ flux through voltage-gated channels with flow cessation in pulmonary microvascular endothelial cells. Microcirculation. 2004;11(6):517-526.

9. Zhou C, et al. Alpha1G T-type calcium channel selectively regulates P-selectin surface expression in pulmonary capillary endothelium. Am J Physiol Lung Cell Mol Physiol. 2010;299(1):L86-L97.

\title{
The bitter taste of infection
}

\author{
Alice Prince \\ Department of Pediatrics, Columbia University, New York, New York, USA.
}

\begin{abstract}
The human innate immune response to pathogens is complex, and it has been difficult to establish the contribution of epithelial signaling in the prevention of upper respiratory tract infection. The prevalence of chronic sinusitis in the absence of systemic immune defects indicates that there may be local defects in innate immunity associated with such mucosal infections. In this issue of the JCI, Cohen and colleagues investigate the role of the bitter taste receptors in airway epithelial cells, and find that these are critical to sensing the presence of invading pathogens.
\end{abstract}

The participation of respiratory mucosal epithelial cells in innate immune defense has been increasingly appreciated. Not only do airway cells express the full complement

Conflict of interest: The author has declared that no conflict of interest exists.

Citation for this article: J Clin Invest. 2012; 122(11):3847-3849. doi:10.1172/JCI66182. of pattern recognition receptors and corresponding adaptor proteins to signal the recruitment of professional immune cells in response to perceived infection, they also participate directly in pathogen eradication. Mucociliary clearance is activated in response to bacterial components, and bacterial killing is mediated through epithelial production of NO and antimicrobial pep- tides. Although major defects in ciliary function (e.g., Kartagener syndrome) are clearly associated with increased respiratory infection rates, more subtle epithelial abnormalities that might be important in susceptibility to common conditions such as chronic sinus infection have not been fully characterized. Mutations in cystic fibrosis transmembrane conductance regulator (CFTR) that do not cause cystic fibrosis have been associated with chronic rhinosinusitis, although the specific pathogenetic mechanisms involved have not been determined (1). Given the complexity of the human innate immune response to pathogens, it has been difficult to establish the contribution of epithelial signaling in the prevention of upper respiratory tract infection. Nonetheless, given the 


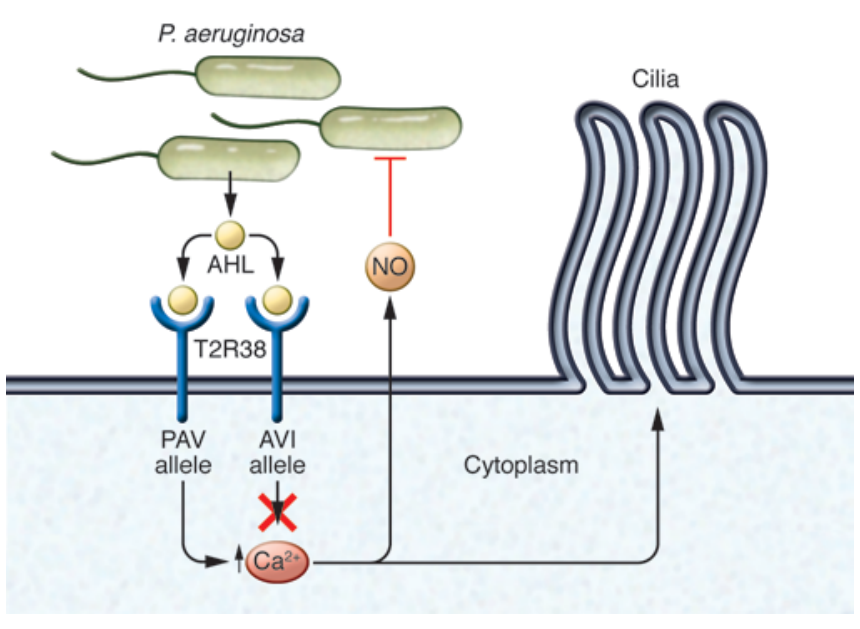

\section{Figure 1}

Bitter taste receptors in innate immune defense. AHLs produced by $P$. aeruginosa are sensed by T2R38, activating $\mathrm{Ca}^{2+}$ signaling, leading to increased ciliary beat frequency, NO production, and bacterial killing. This process prevents colonization of the airway. Individuals harboring the inactive $\mathrm{AVI}$ allele are at greater risk of infection. large number of patients with chronic sinusitis, in the absence of any clinically apparent systemic immune defect, it seems likely that there must be local defects in innate immunity associated with such mucosal infections. In this issue of the JCI, Cohen and coworkers explore unexpected players in innate immune defense: the bitter taste receptors (2).

\section{The taste of toxins}

The bitter taste receptor family (T2Rs) comprises over $25 \mathrm{G}$ protein-coupled receptors that activate PLC- $\beta 2$ and $\mathrm{Ca}^{2+}$ fluxes (3). These proteins recognize a chemically diverse set of bitter-tasting agonists (4). Bitter taste is innate, present in human neonates and in many animals including invertebrates, although it is not universally present in herbivores. The putative function of these receptors is to prevent the consumption of bitter toxins, and there are clear species-specific differences in the relative degrees of bitterness that correlate with various T2R alleles (5). The human genetics of the T2R38 bitter taste receptor has been studied in detail because it confers the ability to perceive the bitter taste of phenylthiocarbamide (PTC) (6). Three nucleotide polymorphisms resulting in 5 haplotypes correlate with the expression of the functional allele of the receptor containing proline, alanine, and valine (PAV/PAV) and a nonfunctional allele containing alanine, valine, and isoleucine (AVI/AVI). The presence of the valine in the third position is suggested to stabilize receptor structure, facilitating activation (7).

The diversity of T2R ligands suggests that functions in addition to bitter taste perception may also be linked to their activation. Among the structures of the agonists for the human bitter taste receptor TAS2R46 are members of the sesquiterpene lactones and similar compounds (4). This group of compounds includes the acylhomoserine lactones (AHLs) produced by $P$. aeruginosa. These AHLs are secreted by bacteria and function in cell-to-cell signaling or quorum sensing that results in the coordinate activation of bacterial genes, including those involved in biofilm formation (8).

As would be expected, the T2Rs are found on the tongue and in the oral mucosae, but in addition they are prominently expressed in the motile cilia and on the surface of airway epithelial cells. T2R activation on ciliated airway epithelial cells induces $\mathrm{Ca}^{2+}$ fluxes, resulting in the stimulation of ciliary beat frequency (9). The function of these receptors present on the proximal airway cells was unclear but was hypothesized to represent a mechanism for the mechanical clearance of noxious airway particles or volatile molecules. The T2Rs have also been identified on airway smooth muscle cells, where they induce $\mathrm{Ca}^{2+}$ fluxes that induce bronchodilation (10), and the utility of this family of receptors as therapeutic targets for bronchodilation in asthma and chronic obstructive pulmonary disease (COPD) had been suggested (10). In addition, given the ability of these receptors to respond to homoserine lactones, it was also postulated that they could function in innate immunity. However, the biologically relevant agonists for the $\mathrm{T} 2 \mathrm{R}$ receptors strategically placed in the respiratory tract were unknown.

\section{Tasting the air}

In the present work, Cohen and coworkers demonstrate that the expression of T2R38 in human respiratory epithelial cells functions to signal the presence of the $P$. aerugi- nosa homoserine lactone (2). Activation of the functional PAV/PAV but not the nonfunctional AVI/AVI receptors results in $\mathrm{Ca}^{2+}$ fluxes, stimulation of ciliary beat frequency, and induction of sufficient epithelial NO production to kill $P$. aeruginosa. Using material from patients referred for sinus surgery, Cohen and colleagues (2) demonstrate that the TSR38 PAV/PAV genotype, as opposed to nonfunctional genotypes, is highly associated with the absence of either Gram negative or, specifically, $P$. aeruginosa sinus infection (Figure 1).

While previous studies hinted at a role for T2Rs in the recognition of bacterial products (9), this study very clearly demonstrates that T2R38 is present not simply to enhance the perception of bitterness or to trigger avoidance of potential toxins, but also to play an important role in host defense. Cohen and coworkers demonstrate the generation of $\mathrm{Ca}^{2+}$ fluxes within 5 seconds of exposure to $P$. aeruginosa AHLs. The T2R38 receptors thus function in the immediate detection of these compounds, acting well before any consequences of TLR or Nod-like receptor signaling would be detectable. Although AHLs are usually associated with $P$. aeruginosa within the context of biofilm formation and established infection, the authors demonstrate that AHLs are expressed even in the very early stages of infection, in amounts sufficient to trigger T2R38 signaling. $P$. aeruginosa mutants lacking AHL production do not trigger T2R38 activation. Thus these receptors function in the prevention of colonization. As colonization of the upper airway generally precedes aspiration of these pathogens and lower respiratory tract infection in susceptible hosts, the $\mathrm{T} 2 \mathrm{R}$ receptors are likely be important in the prevention of pneumonia as well as sinusitis. 


\section{Implications}

The protection mediated by $\mathrm{T} 2 \mathrm{R}$ signaling is likely to function against a large group of potential Gram-negative pathogens. AHLs are highly conserved molecules used by many Gram-negative bacteria to coordinate gene expression (8). Although P. aeruginosa is the best-studied AHL system and arguably the most relevant human pathogen in this group of opportunists, similar signaling systems are widespread, particularly in the marine Vibrios and Aeromonas species, as well as in common opportunists such as Acinetobacter and Burkholderia species $(11,12)$. Thus, T2R signaling would be expected to contribute to innate immune defenses against a number of potential pathogens that are associated with respiratory infection.

Given the interest in identifying genes that confer increased risk or resistance to specific diseases, would the identification of a patient's T2R38 genotype be clinically useful? In those with known increased susceptibility to respiratory infection, such as those with cystic fibrosis or COPD, or even patients in intensive care units expected to require assisted ventilation, Gram-positive as well as Gram-negative infections are fre- quent, and it would be difficult to imagine a scenario in which the T2R genotype would affect therapy that is usually based on bacterial culture results. However, in selected patient groups, such as those with chronic sinusitis, it should be possible to design a prospective study to determine whether the T2R38 genotype can be used to direct preventative antibiotic therapy.

\section{Acknowledgments}

The author's laboratory is supported by NIH grants RO1HL079395 and HL73989.

Address correspondence to: Alice Prince, Department of Pediatrics, Columbia University, 650 West 168th Street, Black Building 418, New York, New York 10032, USA. Phone: 212.305.4193; Fax: 212.305.2284; E-mail: asp7@columbia.edu.

\footnotetext{
1. Wang X, et al. Mutation in the gene responsible for cystic fibrosis and predisposition to chronic rhinosinusitis in the general population. JAMA. 2000;284(14):1814-1819.

2. Lee RJ, et al. T2R38 taste receptor polymorphisms underlie susceptibility to upper respiratory infection. J Clin Invest. 2012;122(11):4145-4159.

3. Chandrashekar J, et al. T2Rs function as bitter taste receptors. Cell. 2000;100:703-714.

4. Brockhoff A, Behrens M, Massarotti A, Appendino G, Meyerhof W. Broad tuning of the human bitter
}

taste receptor hTAS2R46 to various sesquiterpene lactones, clerodane and labdane diterpenoids, strychnine, and denatonium. J Agric Food Chem. 2007;55(15):6236-6243

5. Meyerhof W. Elucidation of mammalian bitter taste. Rev Physiol Biochem Pharmacol. 2005;154:37-109.

6. Kim UK, Jorgenson E, Coon H, Leppert M, Risch $\mathrm{N}$, Drayna D. Positional cloning of the human quantitative trait locus underlying taste sensitivity to phenylthiocarbamide. Science. 2003; 299(5610):1221-1225.

7. Tan J, Abrol R, Trzaskowski B, Goddard WA 3rd. 3D structure prediction of TAS2R38 bitter receptors bound to agonists phenylthiocarbamide (PTC) and 6-n-Propylthiouracil (PROP). J Chem Inf Model. 2012;52(7):1875-1885.

8. Davies DG, et al. The involvement of cell-to-cell signals in the development of a bacterial biofilm. Science. 1998;280(5361):295-298.

9. Shah AS, Ben-Shahar Y, Moninger TO, Kline JN, Welsh MJ. Motile cilia of human airway epithelia are chemosensory. Science. 2009;325(5944):1131-1134.

10. Deshpande D, et al. Bitter taste receptors on airway smooth muscle bronchodilate by localized calcium signaling and reverse obstruction. Nat Med. 2010;16(11):1299-1304.

11. Anbazhagan D, Mansor M, Yan GO, Md Yusof MY, Hassan H, Sekaran SD. Detection of quorum sensing signal molecules and identification of an autoinducer synthase gene among biofilm forming clinical isolates of Acinetobacter spp. PloS One. 2012; 7(7):e36696.

12. Galloway WR, Hodgkinson JT, Bowden S, Welch M, Spring DR. Applications of small molecule activators and inhibitors of quorum sensing in Gram-negative bacteria [published online ahead of print July 6, 2012]. Trends Microbiol. doi:10.1016/ j.tim.2012.06.003

\section{Turning a blind eye to anti-VEGF toxicities}

\section{Susan E. Quaggin}

The Samuel Lunenfeld Research Institute, Mount Sinai Hospital, and Division of Nephrology, St. Michael's Hospital, University of Toronto, Toronto, Ontario, Canada.

\begin{abstract}
Excessive blood vessel growth is a key feature of many retinal diseases, and recently, anti-VEGF therapy has been successfully applied to treat neovascular age-related macular degeneration (AMD), diabetic macular edema, and retinal vein occlusion. In this issue of the JCI, Kurihara et al. reveal an essential role of Vegfa in maintaining choroid vasculature and cone photoreceptors, critical for central and color vision. Their findings suggest that therapeutic approaches to blocking VEGF signaling in retinal diseases might have unexpected detrimental side effects and that the development of alternative strategies might be necessary.
\end{abstract}

Anti-VEGF agents are a rapidly expanding group of therapeutic compounds developed to target pathologic angiogenesis and excessive vascular permeability in cancers and other diseases. They act by blocking the action of the VEGFA ligand or its receptors. Currently, more

Conflict of interest: The author has declared that no conflict of interest exists.

Citation for this article: $J$ Clin Invest. 2012 122(11):3849-3851. doi:10.1172/JCI65509. than 1500 clinical trials of anti-VEGF receptors are underway. The FDA has already approved anti-VEGF agents for the treatment of solid tumors, including colon, breast, and renal cell carcinoma. A number of eye diseases are also characterized by excessive blood vessel growth and permeability, including neovascular agerelated macular degeneration (AMD), diabetic retinopathy, and retinal vein occlusion (1-6). In a randomized clinical trial, patients with AMD who were treat- ed with intravitreal injections of ranibizumab (Lucentis), which inhibits VEGF ligand binding to its receptor, exhibited improvement in best corrected visual acuity (BCVA) at 12 and 24 months, compared with sham-injected controls (6). These results provided the rationale to consider using these therapies in other retinal diseases characterized by enhanced vascular permeability, such as diabetic retinopathy. In an open-label pilot study in ten patients with diabetic macular edema (DME), multiple intravitreal injections of ranibizumab reduced retinal thickness and improved BCVA at three months (7). Since this original study, seven clinical studies demonstrating positive results for DME have been published (3). While these results are very encouraging and offer potential therapeutic value to many patients, there may be reason for guarded optimism based 